\title{
Modelos de formación a lo largo de toda la vida y la «sociedad del conocimiento» en Europa
}

\author{
Andy Green
}

University of London. Institute of Education

andy.green@ioe.ac.uk

Recibido: 01-05-2009

Aceptado: 25-05-2010

\section{Resumen}

La pregunta central que se aborda en este artículo es: «¿Qué tipo de formación a lo largo de toda la vida puede facilitar conseguir una "economía del conocimiento" competitiva y dinámica que también sea una "sociedad del conocimiento" con altos niveles de cohesión social?». El presente artículo trata de examinar estas cuestiones mediante el análisis de los diferentes modelos nacionales y regionales de la "economía del conocimiento" y la «sociedad del conocimiento» en Europa, así como la contribución de la formación a lo largo de toda la vida en cada uno de ellos. Para hacerlo, trata de manera comparada, en primer lugar, de los diferentes modelos de «economía del conocimiento» y de «sociedad del conocimiento» y, en segundo lugar, de su relación con los sistemas de formación a lo largo de toda la vida.

Palabras clave: formación a lo largo de toda la vida; competitividad; cohesión social.

\begin{abstract}
Models of Lifelong Learning and the 'Knowledge Society' in Europe
The main question that informs this article is: which system of lifelong learning is able to facilitate a competitive and dynamic "knowledge economy", while simultaneously also promoting a "knowledge society" with high levels of social cohesion? This article attempts to examine these questions by means of an analysis of national and regional models of the "knowledge economy" and the "knowledge society" in Europe, and the contribution of lifelong learning programmes to each of these models. First, we compare the different models of the "knowledge economy" and the "knowledge society" and, second, their relationship with systems of lifelong learning.
\end{abstract}

Key words: lifelong learning; competitiveness; social cohesion.

* Traducción al español a cargo de Beatriz Krayenbühl Gusi 


\section{Sumario}

Modelos de economía del conocimiento

y sociedad del conocimiento

Sistemas de formación a lo largo de toda la vida y modelos de la economía del conocimiento (sociedad del conocimiento)
¿Qué procesos contribuyen a conseguir los efectos económicos y sociales en cada modelo?

Conclusiones

Referencias bibliográficas

Con frecuencia, se considera la formación a lo largo de toda la vida como una clave para la competitividad económica y la cohesión social. Para la Comisión Europea, resulta fundamental en el objetivo de Lisboa para «una economía competitiva y basada en el conocimiento [...] con más y mejor empleo y una mayor cohesión social» (CE, 2004; Leney et al., 2004; Green et al., 2006). Pero, ¿hasta qué punto es posible conseguir una «economía del conocimiento» competitiva y dinámica que también sea una «sociedad del conocimiento» con altos niveles de cohesión social? Y si así fuese, ¿qué tipo de formación a lo largo de toda la vida tendría mayores posibilidades de fomentarla, y combinada con qué políticas en otros ámbitos económicos y sociales? El presente artículo trata de examinar estas cuestiones mediante el análisis de los diferentes modelos nacionales y regionales de la «economía del conocimiento» y la «sociedad del conocimiento» en Europa, así como la contribución de la formación a lo largo de toda la vida en cada uno de ellos.

\section{Modelos de economía del conocimiento y sociedad del conocimiento}

Los debates sobre la economía del conocimiento frecuentemente se centran en dos modelos que se consideran las principales alternativas para las economías competitivas en un mundo globalizado. En los debates políticos, a menudo se habla de ellos como del modelo «neoliberal» o «anglosajón», representado por Estados Unidos y algunos otros países anglófonos, y del modelo «social de mercado", representado por varios países del norte de Europa, en particular, por Alemania. A menudo, la literatura comparada global sobre la economía política y las competencias presenta un dualismo similar pero con diferentes modelos regionales que se considera representan las antinomias básicas (Hall and Soskice, 2001; Lloyd y Payne, 2004; Crouch and Streeck, 2006). Hutton (1995) contrasta el modelo del "accionista» (shareholder) del capitalismo angloamericano con el modelo de los "participantes» (stakeholder), que se considera está representado por Alemania, Japón y algunos otros estados del norte de Europa. Albert (1993) compara el modelo de mercado del capitalismo angloamericano, con su cultura de contratar y despedir, de absorción de empresas considerándolas como «mercancias», con el capitalismo del «modelo del Rin» de Alemania, Austria y Suiza, en el que las empresas son consideradas "comunidades» y el partenariado social se interpone al poder de los accionistas. Más recientemente, Dore (2000) compara el «capitalismo del mercado bursátil» 
de Estados Unidos y otras economías anglosajonas con lo que él denomina el capitalismo del «bienestar social» o «relacional» de Alemania y Japón. En este artículo, se realizan una serie de comparaciones en base a contrastar un conjunto de factores similares en distintos ejemplos nacionales y sus efectos.

Se dice que las economías de accionistas (del mercado bursátil) dan primacía a los mecanismos de mercado y a los derechos preponderantes de los inversores; las economías de participantes (asistencia social) equilibran estos derechos de los accionistas con los de otras partes interesadas en la empresa y la sociedad. Con ello, moderan los efectos de los mercados mediante la regulación y la intermediación con grupos de interés entre organismos sociales asociados y coordinados u otros intereses (Streeck y Schmitter, 1991; Streeck, 1992; Hutton, 2002). Se dice que, en el modelo de los accionistas, la innovación y la competitividad se consiguen a través de mercados laborales flexibles, una regulación suave, altos índices de empleo, largas jornadas laborales y bajos índices de gasto social. Se asegura que estos factores fomentan una alta productividad y un rápido crecimiento, pero el precio a pagar por ello puede ser menos servicios públicos, una mayor desigualdad y bajos niveles de cohesión social. En el modelo del participante o modelo social, el gasto social es más elevado y existe una mayor regulación del mercado laboral y otros mercados, lo que tiende a mejorar los servicios públicos y a fomentar la igualdad y la cohesión social. No obstante, las regulaciones, los altos costes sociales y unos índices más bajos de empleo pueden disminuir la productividad general y el crecimiento. Cada uno de estos modelos de competitividad ha sido asociado a diferentes estrategias de formación de competencias (Brown et al., 2001; Crouch et al., 1999.)

De acuerdo con este modelo bastante binario que nos proporcionan algunos economistas políticos, los encargados de formular políticas a menudo acaban considerando la relación entre estos modelos de competitividad económica como si estuviera caracterizada por una serie de «tomas y dacas» (nota del traductor: en el original, trade-offs), en los que los beneficios mutuamente incompatibles se tienen que contraponer - creación de empleo y bajo desempleo contra igualdad de ingresos y calidad de empleo; largas jornadas laborales y sueldos altos contra tiempo de ocio y calidad de vida; innovación y dinamismo económico contra estandarización de garantías sociales y medioambientales.

Sin embargo, la investigación sobre las corrientes recientes de las economías occidentales sugiere que hay que repensar los diversos modelos binarios que enfrentan competitividad económica y cohesión social, y que existen modelos posibles de la sociedad del conocimiento que se acercan a la combinación de ambos. Éstos han sido representados claramente en los últimos años por los estados nórdicos, y también, en algunos aspectos, por Holanda. En la mayor parte de los indicadores que examinaremos más adelante, estos países tienen un alto rendimiento, no sólo en la productividad general, sino también en los resultados sociales.

Podemos analizar las diferencias de estos tres modelos y los países que representan examinando los diversos componentes de la productividad general o PIB per cápita, que incluye la productividad laboral (resultados por hora), los índices de empleo y el promedio de horas trabajadas. De Mooij y Tang (2003) 
lo hacen en términos de la dimensión de la diferencia existente respecto a Estados Unidos en varios estados europeos en cada una de estas medidas. Lo que su análisis demuestra es que, mientras que Estados Unidos aún mantiene la primera posición en términos de PIB por cápita por delante de todos los estados europeos excepto Luxemburgo, la dimensión de la brecha varía sustancialmente de un estado a otro y refleja diversas combinaciones de puntos fuertes y débiles respecto a los diversos componentes de la productividad. En la figura 1, adoptamos un enfoque similar, pero hemos incluido, siempre que ha sido posible, las cifras para todos los estados europeos en el mismo año 2003.

La figura 1 muestra que, en el año 2003, todos los países europeos, incluyendo aquellos con una mayor productividad laboral, se quedaban rezagados en lo que respecta al PIB per cápita (excepto Luxemburgo). Diferentes grupos de países quedaron por detrás por diversas razones. Los países más rezagados respecto a Estados Unidos en PIB per cápita se hallaban en Europa central o en el sur de Europa. Entre éstos, encontramos a Grecia, Portugal, España y la República Checa, que estaba a la zaga de Estados Unidos con un porcentaje de 35 a 60 puntos, y, aún más lejos, Turquía, Hungría y Polonia, con más de 60 puntos respecto a Estados Unidos. La baja productividad laboral es la principal causa de esta diferencia en todos los casos. Sin embargo, esta situación se agravaba por índices de empleo considerablemente más bajos.

No obstante, la baja productividad laboral no era necesariamente la principal causa de la distancia por detrás de Estados Unidos en otros estados euro-

Figura 1. PIB/cápita, PIB/hora, horas trabajadas y participación de la mano de obra en países europeos seleccionados como porcentaje de la diferencia con Estados Unidos en 2003.

Porcentaje de diferencia con Estados Unidos

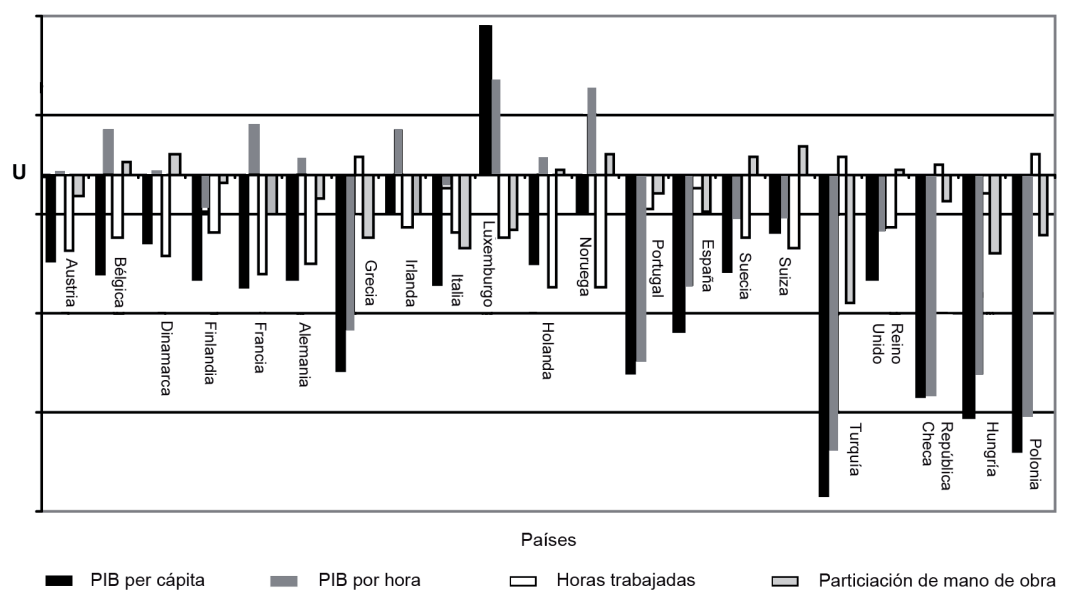

Fuente: Green, A. et al. 2006. Figura 6.1, p. 146 
peos. Austria, Bélgica, Dinamarca, Francia, Alemania, Irlanda, Luxemburgo, Holanda y Noruega gozaban todos de una productividad laboral más elevada que Estados Unidos, pero todos, excepto Luxemburgo, iban rezagados en el PIB per cápita, con diferencias que iban de un 28,4 por ciento (Francia) a un 9,6 por ciento (Irlanda). Éstos y otros países con una productividad laboral bastante cercana a los niveles de Estados Unidos quedaban rezagados respecto a este país en riqueza global por diversas razones.

Los países del corazón de Europa, lo que incluye aproximadamente a todos los de la Europa Occidental continental, excepto los denominados «estados en consolidación", como España, Portugal y Grecia, todos gozaban de una productividad más elevada que la de Estados Unidos, pero todos, excepto Luxemburgo, van rezagados respecto a la riqueza, debido a los índices más bajos de empleo y jornadas laborales más cortas, o ambos factores a la vez. Dos grupos emergen. El grupo mayoritario incluye aquellos países con índices de empleo de más de un 5 por ciento por debajo de los niveles de Estados Unidos: Austria ( $-5,5$ por ciento), Francia ( -10 por ciento), Alemania $(-5,9$ por ciento) e Italia ( -18 por ciento). Estos países presentaban unos niveles de PIB per cápita de entre un 22 (Austria) y un 28 por ciento (Francia) por detrás de Estados Unidos. El grupo minoritario incluye dos países (Bélgica y Holanda), cuyos índices de empleo son más altos que los de Estados Unidos, pero que, de todos modos, quedaban por detrás en los niveles de riqueza debido a jornadas laborales substancialmente más cortas. Aunque geográficamente forman parte del corazón de Europa, en algunos aspectos, estos dos países mostraban resultados más parecidos a los de los países nórdicos.

Los dos países anglófonos, el Reino Unido e Irlanda, se ajustaban más al modelo norteamericano que los países centroeuropeos en algunos aspectos, porque tenían jornadas laborales cercanas a los niveles de Estados Unidos, y, en el caso inglés, se gozaba de índices de empleo más altos. Sin embargo, en general, presentaban resultados muy diferentes. Irlanda tiene un PIB per cápita considerablemente más elevado que el Reino Unido y que los países centroeuropeos, dado que su productividad laboral es muy alta, más de un 10 por ciento por encima de los niveles norteamericanos. El Reino Unido alcanzaba un PIB per cápita inferior, aproximadamente al nivel de Alemania, Italia y Francia en el extremo más bajo de los países del centro de Europa, ya que la productividad laboral estaba un 13 por ciento por debajo de los niveles de Estados Unidos y aún más por detrás de la mayor parte de los países del centro de Europa.

Los países nórdicos (Dinamarca, Finlandia, Noruega y Suecia) tenían un modelo diferente de los demás grupos. Presentaban índices de empleo más altos que la mayor parte de los países del centro de Europa; todos ellos, excepto Finlandia, superan a Estados Unidos en esta medida. No obstante, igual que los países del centro de Europa, gozan de jornadas laborales considerablemente más cortas que los países anglófonos, excepto Suecia. En general, los países nórdicos combinaban los puntos fuertes de los países centroeuropeos en cuanto a su elevada productividad laboral (todos ellos superaban al Reino Unido, y todos, excepto Suecia, superaban a Estados Unidos), con los puntos fuertes 
Figura 2. Gasto social público como \% del PIB

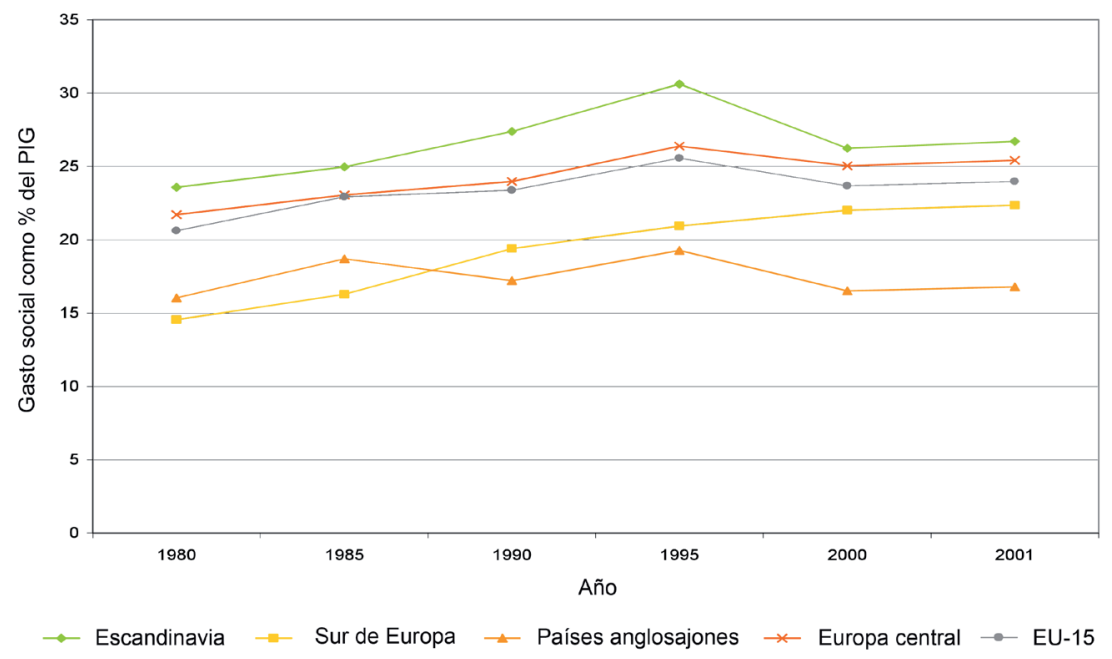

Fuente: OCDE (2004).

de los países anglófonos en altos índices de empleo. Consecuentemente, los resultados generales en PIB por cápita eran más altos en el grupo de países nórdicos que en los estados del centro de Europa o el Reino Unido. Dinamarca, Noruega y Suecia, todos ellos, tenían unos PIB per cápita más altos que el Reino Unido, y Dinamarca y Noruega superaban substancialmente cualquiera de los estados centroeuropeos.

Por supuesto, el sistema de modelos regionales que surge de este análisis no es perfecto. Luxemburgo no encaja en ningún otro grupo; en general, Irlanda es más similar a los países centroeuropeos que el Reino Unido, con una alta productividad laboral y bajos índices de empleo. Holanda e incluso Bélgica están en cierto modo más cerca del grupo nórdico que los países centroeuropeos, en tanto que presentan índices relativamente altos de empleo, así como una elevada productividad laboral. Así pues, podríamos hablar de un grupo nórdico más Holanda y un grupo central europeo más Irlanda; Luxemburgo se mantiene separado de todos los grupos y el Reino Unido sólo forma parte de un grupo con algunos otros países no europeos y anglófonos. No obstante, lo que está claro es que, en cuanto a los elementos que componen la productividad, podemos identificar dos modelos principales y distintos de competitividad en el noroeste de la Europa continental y no uno como normalmente se supone.

Los indicadores sociales también muestran un agrupamiento en términos de las diferentes regiones. La figura 2 muestra los gastos sociales públicos totales como un porcentaje del PIB para varios años entre 1980 y 2001 para países y grupos de países. Los países anglosajones muestran una tendencia relativamente uniforme para esta medida, y para el periodo que empieza en 1990, presenta el nivel medio más bajo de gasto social como proporción del PIB. 
Figura 3. Desigualdad de ingresos

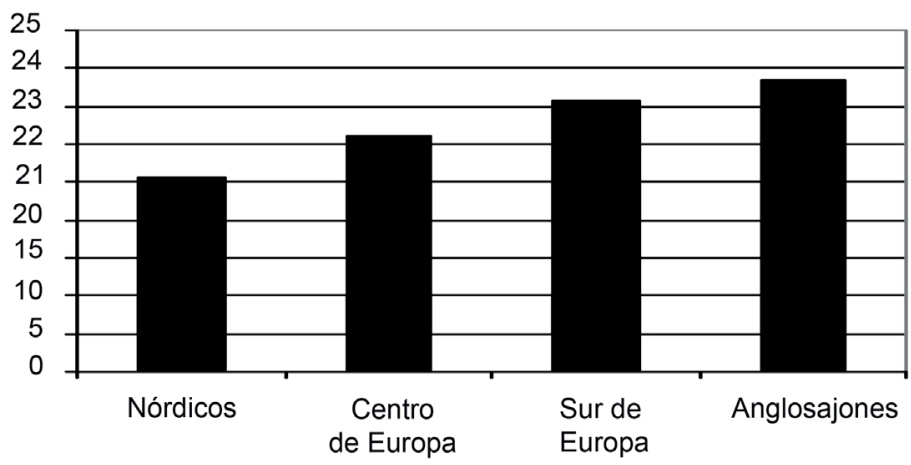

Fuente: World Bank World Development Report 2005.

Los países del sur de Europa empiezan en 1980 con un nivel medio más bajo que los demás grupos, pero presentan un índice más rápido de crecimiento y superan a los países anglosajones a finales de la década de 1980. Los países del centro de Europa tienen un nivel medio creciente de gastos en relación con el PIB, que empieza en cierto modo más alto que la media para los países del sur de Europa y los países anglosajones, y se mantiene así hasta el final del periodo de referencia. El nivel medio más alto de gasto en relación con el PIB se encuentra en los países escandinavos. Éste empieza más alto en 1980 que en cualquier otro grupo y aumenta más rápidamente hasta mediados de la década de 1990. Después, disminuye bastante bruscamente, pero se mantiene más alto de promedio que en cualquiera de los otros grupos. Las fronteras regionales de los tres grupos del norte de Europa se ajustan rigurosamente a las tipologías utilizadas en el estudio de Esping-Andersen de 1990, The Three Worlds of Welfare Capitalism, en el que distinguía entre países anglosajones liberales, países cristianodemócratas corporativistas del centro de Europa y países escandinavos socialdemócratas. Evidentemente, los niveles relativos de gasto social que caracterizan a cada grupo también son consistentes con su análisis original de los niveles de universalismo de la asistencia social atribuible a cada modelo.

La desigualdad en los ingresos muestra los mismos modelos de variación regional. Como podemos ver en la figura 3, existen diferencias notables en los niveles de desigualdad en los ingresos, utilizando la medida índice de Gini para cada uno de los grupos de países. El grupo de países anglosajones presenta el nivel medio más alto de desigualdad, seguido por el grupo de países del sur de Europa. De promedio, los países centroeuropeos son bastante más iguales que estos dos grupos. Pero los cuatro estados escandinavos son los que tienen los niveles más bajos de desigualdad, y ello por un margen bastante sustancial. Los países de cada uno de los grupos están bastante 
estrechamente agrupados en términos de la medida: los países escandinavos tienen coeficientes de Gini de entre 24,7 y 26,9; el grupo de países centroeuropeos, entre 28,3 y 33,1; el grupo del sur de Europa, entre 32,5 y 38,5, y el grupo anglosajón entre 36 y 40,8. Existen solapamientos en los extremos superior e inferior para los países centroeuropeos y los del sur de Europa, y entre el sur de Europa y los anglosajones, pero, en general, los grupos aún son relativamente distintos.

A menudo, las medidas de cohesión social también se agrupan a lo largo de las fronteras regionales. Las investigaciones de Green y Preston (2001) y Green, Preston y Janmaat (2006) sobre las variaciones transnacionales de la cohesión social mostraban unas fuertes correlaciones transversales entre conjuntos de países en cuanto a igualdad de ingresos, igualdad en educación y una medida combinada de cohesión social que incluía a los valores de cada país para "confianza en los demás», "confianza en las instituciones» y "cooperación cívica» (a partir del World Values Survey), e, inversamente, para delitos con violencia (según datos de la Interpol). El análisis estadístico subsiguiente, en el que se utilizaban los datos de series temporales internacionales para una gama más amplia de variables discretas de cohesión social (Green et al., 2006), confirmó las relaciones. Los cambios en los niveles de desigualdad en la educación están relacionados negativamente con cambios en las libertades políticas y civiles y la confianza, y, de forma positiva, con la intranquilidad y la delincuencia. En cada análisis, aparecen en los datos modelos regionales fuertes, y los países nórdicos presentan los valores más altos para las medidas de igualdad de ingresos, igualdad en la educación y cohesión social.

Los diversos datos representados anteriormente relativos a las características económicas y sociales parecen sugerir un modelo de agrupamiento regional con similitudes considerables entre países en cada grupo en la mayor parte de indicadores, y notables diferencias entre los promedios en cada medida para los países de cada grupo. Ciertamente, el agrupamiento por países se podría ajustar en el margen en varias medidas, pero el modelo general sugiere que existe algún sentido en clasificarlos en los cuatro grupos regionales, denominados «escandinavos», «centroeuropeos», «sur de Europa» y «anglosajones», a pesar de que, dado que sólo Estados Unidos y el Reino Unido están muy cercanos en varias medidas, sería mejor denominar los últimos como angloamericanos. Por lo general, los modelos confirman las amplias diferencias en las características que los modelos de política económíca intentan captar, y los países que se considera representan estos modelos se ajustan ampliamente a los grupos regionales adoptados aquí. El análisis de los datos transnacionales también respalda con fuerza la noción de que deberíamos tratar a los países nórdicos como un grupo separado de los países centroeuropeos. En particular, los países escandinavos pueden distinguirse de la mayor parte de países centroeuropeos en que presentan índices de empleo más altos, un gasto social más elevado en relación con el PIB, bajos niveles de desigualdad de ingresos y niveles más altos de cohesión social. Holanda se encuentra en algún lugar entre los dos grupos en la mayor parte de estas medidas. 
Sistemas de formación a lo largo de toda la vida y modelos de la economía del conocimiento (sociedad del conocimiento)

Los sistemas de formación a lo largo de toda la vida en Europa varían considerablemente de un país a otro, pero también existe un agrupamiento considerable que nos permite identificar una serie de modelos regionales y culturales que comparten características similares (Green et al., 2006). En general, los países del sur de Europa tienen sistemas unificados de escuelas públicas para la educación obligatoria, como la mayor parte de países occidentales europeos, pero, generalmente, se distinguen por tener sistemas de aprendizaje débiles y altos niveles de centralización administrativa a nivel gubernamental o regional. Los países germanófonos y los países próximos a Alemania, como Bélgica y Luxemburgo, también tienen sistemas bastante federalizados, pero son los únicos que mantienen la escolarización secundaria selectiva. Los países anglófonos tienen sistemas educativos formalmente unificados, pero un grado de diversidad escolar y una gama de posibilidades para escoger que minan los objetivos de mezcla social de la escolarización global. Por otra parte, los países nórdicos tienen los sistemas educativos unificados más absolutamente desarrollados, con una escolarización no selectiva basada en una escuela unificada de barrio que incluye alumnos con diferentes niveles de capacidad. ¿¿De qué forma se articulan estos sistemas de formación a lo largo de toda la vida regionalmente selectivos con las diversas formas de economía del conocimiento y la sociedad del conocimiento que hemos identificado en las distintas regiones? Podríamos empezar por los resultados que producen en relación con las competencias.

Un argumento tradicional respecto a la formación de competencias y la competitividad económica (Crouch et al., 1999; Brown et al., 2001) es que los diferentes sistemas de formación de competencias tienden a adecuarse a las necesidades de competencias de las economías nacionales y de sus diversos sectores, bien sea satisfaciendo sus necesidades o, desde otra perspectiva, reproduciendo sus patologías (Finegold y Soskice, 1988). Se considera que los sistemas de formación de competencias en el Reino Unido y en los Estados Unidos producen una mano de obra altamente cualificada y una mano de obra poco cualificada excepcionalmente polarizadas. Éstas incluyen una abundante élite altamente cualificada que satisface las necesidades de los sectores que requieren un elevado nivel de conocimientos y personal altamente cualificado para la producción y los servicios de alto valor añadido, como el diseño, los medios de comunicación, la industria farmacéutica, las TI, la ingeniería aérea y de defensa, etc. Por otra parte, la abundante mano de obra poco cualificada satisface las necesidades de los sectores que compiten en costes y eficiencia, donde es necesaria una mano de obra barata y flexible. Otros sectores, como la banca y la industria química, parecen requerir de sus empleados una serie de competencias altamente polarizadas y, por lo tanto, también se puede decir que están bien servidos por los sistemas de formación de competencias. En la comparación típicamente binaria, se considera que países como Alemania y otros estados centroeuropeos compiten económicamente sobre todo a través 
Figura 4. Proporción de empleados en puestos de trabajo altamente cualificados

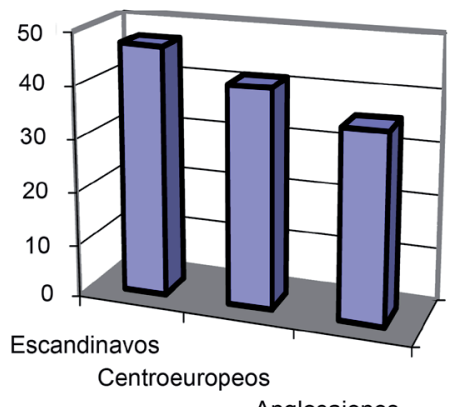

Fuente: OCDE (2000).

de una producción de calidad altamente diversificada en la fabricación, lo que requiere competencias más uniformemente distribuidas y, en particular, un extenso cuerpo de obreros y técnicos especializados y cualificados (Streeck, 1997). En estos países, los sistemas de formación de competencias generalmente lo consiguen, dado que la mayor parte de la mano de obra está cualificada en niveles intermedios, con élites cualificadas y una mano de obra poco cualificada relativamente menores (Brown et al., 2001).

Pero, ¿de qué manera se articulan la formación de competencias y los modelos socioeconómicos en la tipología tripódica de las economías del conocimiento y las sociedades del conocimiento aquí propuestas? Y, ¿cuáles son los mecanismos y las características de los supuestos sistemas regionales de formación a lo largo de toda la vida que fomentan y sostienen las características de los distintos modelos? En este caso, tres aspectos nos incumben. En primer lugar, la proporción general de empleados altamente cualificados que se produce en cada tipo de sistema. En segundo lugar, de qué forma se distribuyen las competencias en la mano de obra. Y, en tercer lugar, hasta qué punto los adultos están implicados en la educación. Cada una de estas características del resultado de la educación está relacionada con las características de los distintos modelos de la economía del conocimiento o sociedad del conocimiento que hemos diferenciado anteriormente.

Primero, para tomar el nivel neto de competencias, los datos de la International Adult Literacy Survey (IALS) (Encuesta Internacional de Alfabetización de Adultos) parecen sugerir que la proporción de empleos altamente cualificados en las economías de los diferentes países es muy consistente, con lo que predecirían los tres modelos regionales de la economía del conocimiento o sociedad del conocimiento. La figura 4 muestra la proporción de trabajadores en empleos altamente cualificados en la época de las encuestas IALS llevadas a cabo entre 1994 y 1998. Dinamarca, Finlandia, Noruega y Suecia aparecen en el estudio y forman un grupo de países con la proporción media más alta de empleos altamente cualificados. Los países del centro de Europa están 
Figura 5. Puntuaciones en alfabetismo de prosa, documental y cuantitativo

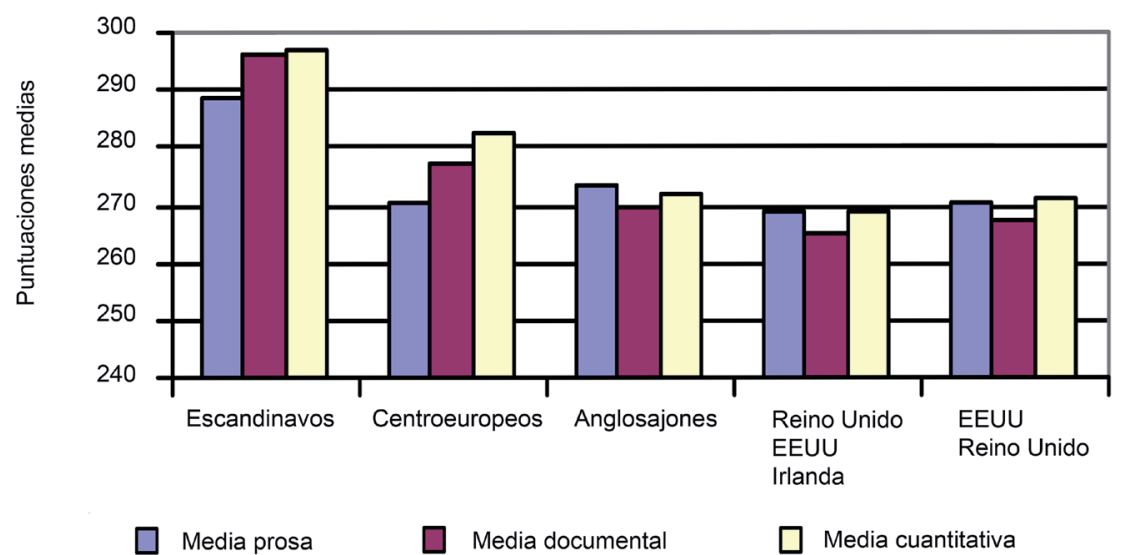

Fuente: OCDE (2000).

representados aquí por Austria, Alemania, Holanda y Suiza, que, de media, presentan una proporción bastante más baja de empleos altamente cualificados. Por otra parte, están los países anglosajones, que incluyen Australia, Canadá, Irlanda, Nueva Zelanda, el Reino Unido y Estados Unidos, y muestran la proporción más baja de empleos altamente cualificados. Las distribuciones regionales son, por lo tanto, consistentes con el modelo tripódico de sociedades del conocimiento o economías del conocimiento que predice élites cualificadas en los países anglosajones, un nivel general superior de competencias en la alta productividad de la mano de obra en los países centroeuropeos y un nivel en cierto modo aún más alto en los aún más igualitarios países nórdicos.

El análisis de la OCDE sobre los datos de las IALS muestra que existe una correlación significativa entre los niveles de alfabetización media por país y la medida principal de competitividad económica: PIB per cápita (OECD, 2000: 80). También existe una fuerte correlación transnacional entre los totales en los niveles de alfabetización y la proporción de empleados en puestos altamente cualificados que se considera incluyen legisladores, altos funcionarios y gerentes, profesionales y profesionales colegiados. En cuanto a los niveles de rendimiento, las IALS también muestran que existen modelos regionales muy claros, por lo menos con respecto a los países nórdicos y anglófonos que pertenecen al único grupo regional y cultural suficientemente bien representado para poder formular una opinión.

La figura 5 muestra las puntuaciones medias para cada país calculadas por grupo de países. Los países escandinavos presentan la puntuación media en alfabetismo de prosa, documental y cuantitativo. Los países centroeuropeos promedian más alto que los países anglófonos en alfabetismo documental y cuantitativo, pero más bajo en alfabetismo de prosa. El grupo formado por Estados Unidos, el Reino Unido e Irlanda obtiene resultados similares al grupo 
Figura 6. Desviación estándar de puntuaciones por grupos de países

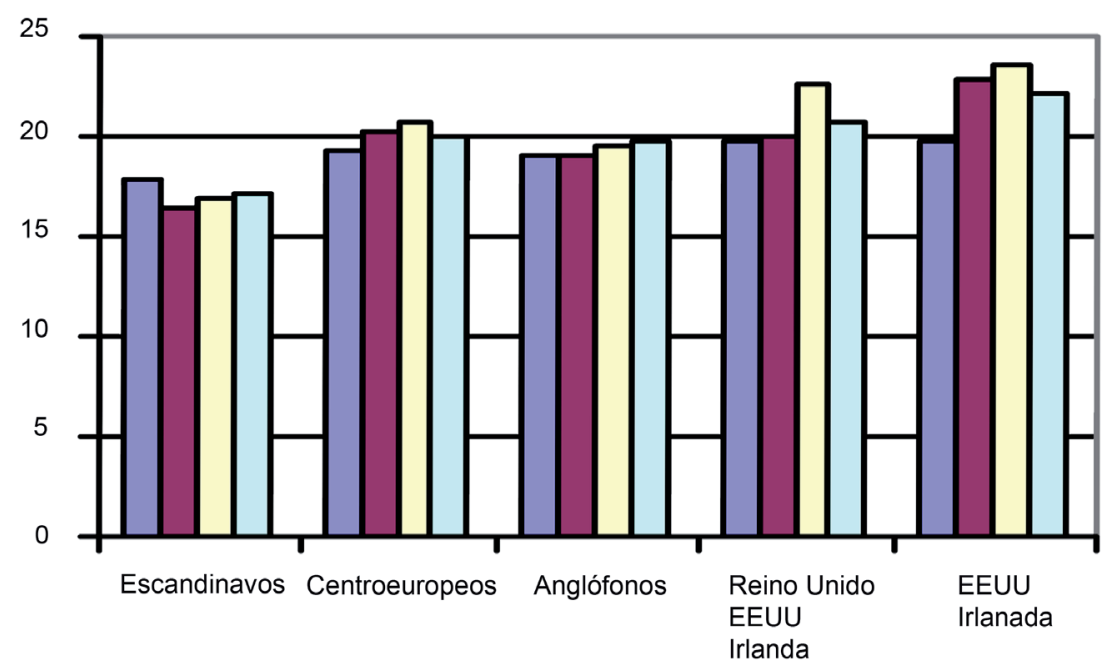

$\square$ Prosa DE $\square$ Documental DE $\square$ Cuantitativo DE $\square$ Media DE

Fuente: Green, A. et al. 2006. Figura 6.10, p. 163.

del Reino Unido y Estados Unidos, y ambos obtienen puntuaciones medias más bajas en todas las medidas que los otros grupos de países de la muestra.

Respondiendo a la pregunta de la igualdad en la educación y la distribución de competencias, tenemos ahora una evidencia bastante clara a partir de los estudios IALS y PISA sobre la forma en que podemos comparar las distintas regiones en cuanto a la distribución de competencias y la igualdad en la educación.

La figura 6 muestra las diferencias entre los grupos de países en términos de la dispersión de las puntuaciones en las tres medidas en las IALS, utilizando las desviaciones estándar. Como podemos ver, los países escandinavos presentan el nivel medio de dispersión más bajo en las tres medidas, y, en general, en las tres medidas combinadas. En conjunto, los países anglófonos presentan niveles de dispersión más elevados que los de los países escandinavos, pero similares a aquellos del grupo de países centroeuropeos. No obstante, las puntuaciones para el Reino Unido y Estados Unidos están más dispersas que en los países anglófonos en conjunto, y son considerablemente más altas que las de cualquier otro grupo de países. Los altos niveles de desigualdad en los resultados de los estados anglófonos con relación a los niveles más bajos de los estados nórdicos también se reflejan en los gradientes socioeconómicos que muestran el impacto de la educación de los padres sobre los resultados individuales (OCDE, 2000: 30). 
Figura 7. Variación media en ciencias por grupos de países en PISA 2006

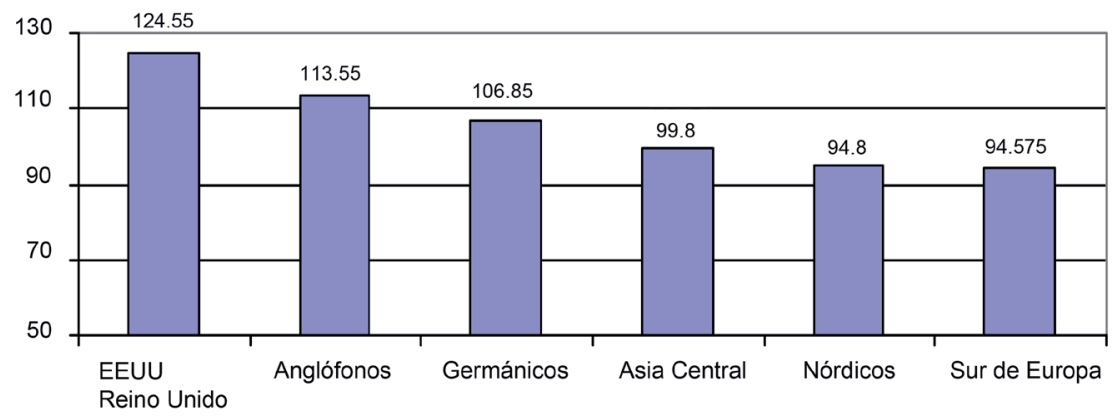

Fuente: OCDE, 2007, 2. Cuadro S2c, p. 250.

La figura 7 muestra la variación en los resultados en ciencias del estudio PISA de 2006, expresada como porcentaje de la variación media en puntuaciones en ciencias en todos los países de la OCDE (cuadro 4.1: 96; OCDE, 2007, vol. 2). La figura 7 presenta las medias de los grupos de países específicos. La dispersión de competencias más amplia se encuentra en el grupo de países anglófonos y en Alemania y países próximos a Alemania. El grupo anglófono presenta la variación media más alta. En este grupo, Canadá $(96,4)$ e Irlanda $(97,5)$ presentan una variación algo más baja, pero la dispersión de competencias es muy amplia en Nueva Zelanda $(125,2)$, Estados Unidos $(124,7)$ y Reino Unido $(124,4)^{1}$. En el grupo «germánico» de países, la variación se halla por encima de la media en todos los países excepto Holanda (Austria $(104,3)$; Bélgica $(108,0)$; Alemania (110,4); Luxemburgo $(103,2)$; Holanda $(99,1)$, y Suiza $(109,6))$. Los grupos más similares son los nórdicos (Dinamarca $(95,6)$; Finlandia $(81,4)$; Islandia $(101,2)$; Noruega $(98,9)$, y Suecia $(96,3))$, así como los países del sur de Europa (Grecia (93,9); Italia $(100,8)$; España $(90,8)$, y Portugal $(87,2)$ ).

Los modelos anteriores sugieren afinidades regionales en cuanto a los niveles de igualdad en la educación. Los países nórdicos forman un grupo bastante bien definido, con niveles en general bastante bajos de desigualdad. Los países anglófonos, excepto Irlanda del Sur y Canadá, forman otro, con niveles de desigualdad muy elevados. Alemania, Bélgica y Suiza, que representan el corazón de Europa en estas muestras, también presentan niveles bastante elevados de desigualdad, aunque Alemania resulta ser más igualitaria en las medidas de competencias en los adultos que en los jóvenes de quince años, posiblemente debido a los efectos mitigadores sobre las bajas competencias experimentados por los jóvenes que pasan por el sistema de aprendizaje. Una investigación reciente de Blanden, Gregg y Machin (2005) también avala este modelo regio-

1. En el Reino Unido, la variación medida por la desviación estándar más alta se halla en Irlanda del Norte (DE: 113), seguida por Inglaterra (107), Gales (102) y Escocia (100): Ver OCDE, 2007, 2, cuadro S2c, vol. 2, p. 250. 
Figura 8. Participación de las personas de 16 a 65 años en la formación de adultos en el año anterior. 1994-1998

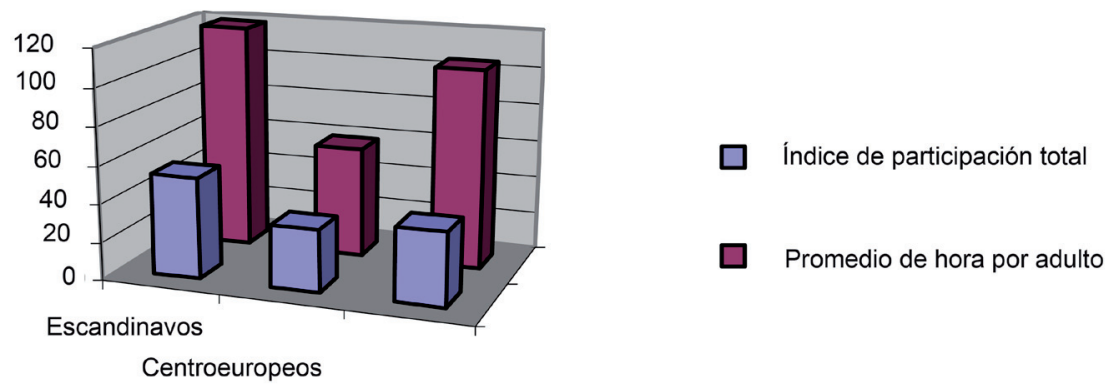

Anglosanjones

Fuente: OCDE (2000).

nal de desigualdad. Utilizando datos longitudinales para las cohortes nacidas en los años 1950 y 1970, se encuentran con que la movilidad intergeneracional en Gran Bretaña y Estados Unidos es sustancialmente inferior a la de Canadá y los países nórdicos. Alemania está situada aparentemente en algún lugar entre los dos grupos (aunque las dimensiones de la muestra nos obligan a matizar la validez de este último resultado). También observaron que la fuerza del efecto de la herencia social, especialmente en lo que concierne al vínculo entre la renta familiar y la participación y los resultados en la educación superior, se hallaba en el centro de la explicación de la cultura de baja movilidad británica.

Una vez más, el modelo regional en la formación a lo largo de toda la vida muestra una estrecha relación con los modelos regionales de la economía del conocimiento o sociedad del conocimiento. El grupo que tiene el nivel más bajo de desigualdad de ingresos, el de los países nórdicos, es el mismo que el grupo con el nivel más bajo de desigualdad en la educación. De igual modo, el grupo con niveles más altos de desigualdad en la educación (el grupo anglófono, menos quizás Canadá e Irlanda, cuyas clasificaciones son ambiguas) es igualmente el grupo con más altos niveles de desigualdad en la renta.

Una última relación entre los modelos de formación a lo largo de toda la vida y los modelos de la economía del conocimiento o sociedad del conocimiento tiene que ver con la distribución de la formación de adultos. Una afirmación que se ha hecho respecto a la particularidad del modelo nórdico de la sociedad del conocimiento es que consigue altos niveles de empleo, a la vez que bajos niveles de igualdad en la renta. Según el análisis de Mooij y Tang (2003), esto se podría explicar en parte por la prevalencia de las políticas activas de empleo, que, a diferencia de otras formas de intervención del mercado laboral, parecen fomentar la ocupación sin reducir la igualdad salarial. Los datos sobre la participación de los adultos en la educación de la figura 8 parecen confirmar esta explicación. Los índices de participación en la educación de adultos, tanto en índices totales como en promedio de horas, son los 
más altos en el grupo de países escandinavos que combinan altos índices de empleo y una baja desigualdad en los ingresos. Los países anglosajones, que, generalmente, gozan de altos índices de empleo (excepto Irlanda) pero presentan niveles más bajos de igualdad en los ingresos, se sitúan entre los países nórdicos y los centroeuropeos en ambas medidas de participación. Los países centroeuropeos, que presentan índices de empleo substancialmente inferiores, aunque una mayor igualdad de ingresos que los países anglosajones, tienen los índices de participación medios más bajos.

\section{¿Qué procesos contribuyen a conseguir los efectos económicos y sociales en cada modelo?}

El modelo aquí propuesto sugiere una serie de hipótesis en cuanto al papel de la formación a lo largo de toda la vida en el desarrollo de los diferentes tipos de economías del conocimiento o sociedades del conocimiento, que hemos caracterizado como liberal y anglosajona; corporativista y centroeuropea, y socialdemócrata y nórdica.

En primer lugar, la economía del conocimiento o sociedad del conocimiento se define en términos de su productividad general y de sus resultados sociales, que están determinados por la diversidad en la productividad laboral, los índices de empleo, la igualdad salarial, la distribución de las competencias y la socialización. El modelo liberal, anglosajón, de la economía del conocimiento tiende a combinar una productividad laboral moderada con altos índices de empleo y una gran desigualdad en los ingresos, con una productividad general media-alta en el ámbito económico y, en el social, un gasto social moderado y medidas de cohesión social más bajas. El modelo corporativista centroeuropeo de la sociedad del conocimiento combina una alta productividad laboral con índices más bajos de empleo y una más baja desigualdad salarial, que da como resultado una productividad general moderada-alta en el ámbito económico y, en el social, unos resultados más altos de gasto social y de cohesión social. El modelo nórdico combina una productividad laboral más alta con índices de empleo altos y una relativa igualdad salarial, con una alta productividad general en el ámbito económico, y, en el social, un gasto social elevado y una alta cohesión social.

En segundo lugar, la formación a lo largo de toda la vida tiene un papel fundamental en la generación de las causas más directas de los diferentes modelos, en conjunción con, inter alia, los efectos de los sistemas de bienestar y la regulación del mercado laboral. Los sistemas de formación a lo largo de toda la vida producen diferentes tipos de distribución de competencias y modalidades diferentes de socialización que afectan a los índices de empleo, la productividad, la distribución de la renta y la cohesión social, directa o indirectamente. En este caso, pasamos por alto el proceso de socialización y de formación de la identidad, aunque se trata sin duda de un factor determinante en los diferentes sistemas de cohesión social, y nos centramos en los efectos distributivos. Encontramos tres dimensiones de resultados de la formación a lo largo de 
toda la vida que parecen ser cruciales para cada una de las cadenas causales: el resultado general de las competencias en el mercado laboral, la distribución de estas competencias y los índices de participación en la formación de adultos. Hemos identificado tres maneras en que los diferentes resultados de la formación a lo largo de toda la vida pueden tener un impacto en el modelo de economía del conocimiento, aunque éstas no se pueden considerar de forma totalmente individual.

En primer lugar, obviamente, los sistemas de formación a lo largo de toda la vida, que introducen altos niveles promedio de competencias en el mercado laboral, probablemente contribuirán a la alta productividad laboral general, al convertir las competencias en resultados productivos, con la ayuda de la inversión de capital y otros factores de producción. En segundo lugar, los sistemas de formación a lo largo de toda la vida generan diferentes niveles de desigualdad en las distribuciones de competencias que se transmiten al mercado laboral. Estas distribuciones diferentes de competencias, en conjunción con formas distintas de regulación del mercado laboral, afectarán a los niveles de ingresos y a la desigualdad de estatus entre los empleados, que, junto con los efectos redistributivos de los sistemas de bienestar sobre la distribución general de los ingresos de la unidad doméstica, acabarán impactando en los niveles de cohesión social. En tercer lugar, los sistemas de formación a lo largo de toda la vida tendrán su impacto sobre los índices de empleo que afectan tanto a la productividad general como a la cohesión social. En particular, los sistemas de formación a lo largo de toda la vida con altos niveles de participación de los adultos en la educación, especialmente a través de la política activa de empleo, favorecerán altos índices de empleo, que son una manera — aunque parcial— de aumentar la cohesión social a través de la «inclusión social».

Así pues, ¿cuáles son las estructuras y los procesos en los sistemas de formación a lo largo de toda la vida de cada modelo que producen efectos notables?

Los sistemas de formación a lo largo de toda la vida del modelo liberal, anglosajón, tienden, como hemos visto, a producir niveles agregados moderados de competencias en el mercado laboral, con un alto nivel de polarización de competencias entres las élites y la mano de obra poco cualificada. Estos resultados pueden contribuir a conseguir unos niveles medios bastante moderados de productividad laboral, un alto nivel de desigualdad en los ingresos y un bajo nivel de cohesión social. Los niveles agregados moderados de competencias son el producto de una polarización de las competencias, una vez que las altas competencias de las élites han sido equilibradas por las competencias inferiores de la mano de obras menos cualificada (suponiendo que exista un límite al grado en que se pueda elevar el promedio general sin atenuar el extremo inferior); así pues, ello se traduce en las desigualdades en las competencias y en el problema de la «larga cola de personas que no rinden al nivel de su capacidad o al nivel exigido" (nota del traductor: en el original, underachievers) en estos sistemas. ¿Por qué los sistemas del modelo anglosajón producen un nivel tan elevado de desigualdad de competencias? Una respuesta posible se halla en los efectos de sus sistemas de ayuda social, que tienden a contar con una oferta 
escasa de educación preescolar para las personas con bajos ingresos y que hacen poco para ayudar a los ingresos de la familias con ningún sueldo o con sueldos bajos. Tal como numerosas investigaciones demuestran, los niños de familias con sueldos bajos — que, en los países que siguen este modelo, posiblemente sean de forma desproporcionada las familias monoparentales- tienen más posibilidades de rendir por debajo de sus capacidades o del nivel exigido en la educación, especialmente si existe una educación preescolar inadecuada para mitigar los efectos del nivel de capital humano y cultural inferior de estas familias. No obstante, parece probable que en esto también intervenga un efecto del sistema escolar.

Los sistemas escolares de los países anglosajones, y especialmente los del Reino Unido, Estados Unidos y Nueva Zelanda, son notables debido a sus altos niveles de diversidad escolar y de elección, así como por tener sistemas reguladores basados en una competencia de quasi mercado y de gestión escolar local que posiblemente incrementen la diversidad académica. Dado que la diversidad que existe en las escuelas, en la mayoría de casos, parece traducirse en una diversidad media en los estándares escolares, estos sistemas, aunque nominalmente unificados en su estructura, tienden a producir altos niveles de variación en los resultados académicos entre escuelas (OCDE, 2002), lo cual posiblemente aumente la desigualdad general de los resultados, puesto que permanece el resto constante. En la medida en que la opción de la escuela está vinculada a la capacidad económica o a la dotación de capital cultural asociada al empleo y a los ingresos, tales sistemas probablemente incrementarán el grado de determinación del entorno social en los logros escolares, con lo cual se reducirá la movilidad social y aumentará la desigualdad social en la educación. Como hemos visto, esto puede tener efectos negativos sobre la desigualdad en los ingresos y, en última instancia, sobre la cohesión social.

Por otra parte, los sistemas de formación a lo largo de toda la vida de los países anglosajones tienden a proporcionar oportunidades bastante buenas para la formación de adultos, tal como se puede inferir de los índices relativamente altos de participación alcanzada. A pesar de que la experiencia sugiere que tales oportunidades están estrechamente vinculadas a los niveles educativos iniciales - por lo tanto, acentuando de hecho las desigualdades heredadas del sistema escolar (Brown et al., 2001; EC, 1999)—, la existencia de altos índices de formación de adultos, aunque desigualmente distribuidos y especialmente relacionados con la política del mercado laboral activo, probablemente incrementará los índices de empleo mediante un proceso por el cual los trabajadores excluidos tendrán más posibilidades de ser contratados. Los altos índices de empleo reducirán el número de los socialmente excluidos, y, por lo tanto, tendrán probablemente efectos positivos sobre la cohesión social. Sin embargo, en el caso de los países del modelo anglosajón, los efectos pueden ser limitados, debido a que la alta desigualdad salarial, aún en condiciones de casi pleno empleo, puede ser perjudicial para la cohesión social.

Los sistemas de formación a lo largo de toda la vida de los países centroeuropeos parecen actuar de forma distinta. Producen niveles totales de competen- 
cias que son tan altos, cuando no más altos, que los de los países anglosajones, dependiendo del caso de cada país, que tendrán efectos positivos en la productividad laboral. También parecen producir distribuciones de competencias bastante menos dispersas, por lo menos entre la mano de obra adulta, que son propicias a la creación de niveles bastante más altos de igualdad en los ingresos que pueden ser beneficiosos para la cohesión social. No obstante, esto puede parecer paradójico, dado que sus sistemas escolares obligatorios producen resultados muy desiguales. Podemos ofrecer una serie de explicaciones. Los sistemas de escolarización obligatoria realmente producen resultados muy desiguales (excepto en el caso de Francia), ya que, excepto este país, todos los países de este grupo tienen sistemas escolares selectivos y diferenciados que, por su propia naturaleza, intentan producir una diversidad de resultados. Sin embargo, la desigualdad de los resultados medidos en los jóvenes de quince años de edad no se refleja en países como Alemania en la población total adulta. Esto obedece, probablemente, a dos razones: una es que el cambio en los índices de cualificación en un cierto número de estos países ha sido relativamente lento respecto a otros grupos de países; por lo tanto, existe menos variación en los niveles de competencias entre las cohortes en la población adulta. La segunda es que todos estos países, de nuevo excepto Francia, tienen sólidos sistemas de aprendizaje que proporcionan tres años más de educación general (y profesional) a la mayor parte de la gente joven que participa en ellos. Probablemente, esto tenga un efecto sustancial en la mitigación de la variación en los niveles de competencias en alfabetismo y nociones elementales de cálculo aritmético observados en los jóvenes de quince años, aunque, para verificar esta hipótesis, serían necesarias exploraciones complementarias con los jóvenes de dieciocho y diecinueve años. El impacto neto de estos efectos de dos sistemas es un nivel de desigualdad en las competencias adultas más elevado que en los países nórdicos, pero bastante más bajo que en los países anglosajones. Esto está combinado con formas de regulación del mercado laboral que reducen la desigualdad salarial (por ejemplo: en la mayoría de países, existen diversas combinaciones de salarios mínimos, negociación salarial por sectores y fijación de índices salariales para los empleos, determinación conjunta en las empresas, etc.), y ello sirve para reducir la desigualdad salarial general y fomentar la cohesión social.

No obstante, en los países centroeuropeos, tiene lugar otro proceso que puede no ser tan beneficioso para la igualdad general y la cohesión social. La regulación del mercado laboral, en forma de leyes estatutarias sobre la autorización para ejercer y acuerdos sectoriales sobre salarios y cualificaciones para los empleos cualificados, tiene consecuencias adversas en términos de igualdad general, así como efectos beneficiosos para la igualdad en los ingresos. En primer lugar, la fuerte regulación del mercado laboral eleva los costes de los empleadores para la creación de nuevos puestos de trabajo y, por lo tanto, estará relacionada con un paro más elevado. En segundo lugar, dado que los sistemas del mercado laboral sirven para definir la mayor parte de los empleos como empleos cualificados y para reforzar los requisitos de cualificación para el acceso a estos empleos, éstos crean fuertes barreras al trabajo para los no cuali- 
ficados. Mientras que los sistemas de aprendizaje generalmente proporcionan una transición bastante suave al empleo para aquellos que participan en ellos, para aquellos que no pueden conseguir puestos de aprendizaje y que no han adquirido de otra forma títulos de educación general que les ayudarían a obtener un empleo, las perspectivas de trabajo son muy sombrías. Típicamente, la proporción de los que no tienen ni siquiera cualificaciones especializadas (en el nivel 3 ISCED) en estos países es bastante inferior a la de los países anglosajones (Brown et al., 2001). Por otra parte, probablemente, están bastante más desfavorecidos debido a las importantes barreras para conseguir un empleo. Así pues, los países centroeuropeos, con fuertes regulaciones del mercado laboral, así como con unos sistemas de formación a lo largo de toda la vida muy bien definidos, crean algo que se parece a una situación con aspectos negativos y positivos en cuanto a la inclusión social y la igualdad. Alcanzan niveles relativamente altos de igualdad en los ingresos, pero a costa de una división creciente entre el individuo empleado y el individuo en paro, una situación que sólo se atenúa en parte con generosos sistemas de bienestar. Esto podría explicar en parte por qué en muchos casos los niveles de cohesión social no son tan elevados como podría sugerir el alto grado de igualdad en los ingresos.

Finalmente, los países nórdicos parecen mostrar una serie de relaciones bastante diferentes. Producen altos niveles generales y una distribución de competencias en la mano de obra poco dispersa, lo que contribuirá a conseguir los altos niveles de productividad laboral, a la vez que reducirán la desigualdad salarial (Nickel y Layard, 1998). La elevada participación en la formación de adultos fomentará altos índices de empleo que, junto con altos índices de productividad, producen altos niveles de productividad general. Los altos índices de empleo, junto con una escasa desigualdad en los ingresos y los fuertes efectos redistributivos de los sistemas de bienestar, reducirán la desigualdad general y sostendrán la cohesión social.

Los sistemas de formación a lo largo de toda la vida parecen contribuir de dos maneras a conseguir los resultados positivos, tanto en el ámbito económico como en el social. En primer lugar, producen niveles de competencias relativamente similares, tanto en los jóvenes de quince años como en los adultos. Esto puede deberse, en parte, a los efectos de ayuda social de EspingAndersen. Las políticas de protección y regulación laboral reducen la incidencia de familias con poco ingresos, cuyos hijos, estadísticamente, tendrían mayores probabilidades de rendir por debajo de sus capacidades o del nivel exigido en la educación. La educación preescolar universal proporcionada por el estado también facilita una socialización temprana que compensa el efecto de menores aspiraciones y logros en la educación de los niveles inferiores de capital humano y cultural de las familias (Esping-Andersen, 2003). Consiguientemente, el rendimiento por debajo del nivel exigido vinculado a la clase social - $\mathrm{y}$, especialmente en los países anglosajones, a rentas bajas, entorno familiar monoparental - es reducido, con lo cual se incrementa la igualdad general en los resultados en la educación. No obstante, también podría ser muy probable que hubiese algunos efectos del sistema educativo. Lo que es 
notable respecto a los sistemas nórdicos (y, evidentemente, asiáticos) relativamente igualitarios es que todos gozan de sistemas escolares completamente unificados y no selectivos en el sector estatal, con una enseñanza con alumnos de diferentes niveles de capacidad virtualmente en todos ellos, y sectores privados no de élite bastante reducidos en la educación. En el caso nórdico, las estructuras igualitarias están compuestas por la base habitual de admisión a la escuela de los vecinos del barrio. Dado que la escolarización primaria y secundaria inferior está integrada en todas las escuelas que combinan la enseñanza primaria y la secundaria (all-through schools), el problema de elegir escuela a la edad de acceder a la educación secundaria inferior desaparece excepto en el caso de que las familias cambien de zona. Dado que los niveles inferiores de desigualdad en los ingresos en los países nórdicos normalmente representan una diferenciación de clase social bastante reducida entre barrios, en donde las escuelas admiten a los niños que sean del barrio, esto también representará probablemente niveles bastante más bajos de diferenciación social entre escuelas, y, consecuentemente, niveles más bajos de variaciones en los rendimientos de una escuela a otra (OCDE, 2002).

Un segundo efecto se halla en los altos niveles de participación en la formación de adultos que tiene lugar en los países nórdicos. Esto está relacionado en parte con las medidas del mercado laboral activo, que estimulan la reconversión de las competencias de los parados y de las personas que están a punto de quedarse sin trabajo, que pueden tener efectos muy positivos sobre los índices de empleo y, consecuentemente, sobre la inclusión social. También está relacionado con la oferta bastante generalizada de formación general de adultos financiada por el estado (que no es lo menos importante en las escuelas populares de secundaria de día e internados, o Folkeskole), que puede contribuir a la empleabilidad de los adultos, pero que también sirve —o, por lo menos, intenta servir en el ámbito social — de foro para fomentar el espíritu de comunidad, la consciencia política y la cohesión social (Boucher, 1982; Green y Janmaat, 2011; Rubenson, 2002).

Considerados de forma conjunta, los sistemas escolares igualitarios y las amplias oportunidades de formación para los adultos podrían maximizar los efectos potenciales sobre la formación a lo largo de toda la vida a través de la inclusión social (en el mercado laboral), la socialización y la igualación de los ingresos.

\section{Conclusiones}

El análisis que hemos llevado a cabo en este artículo sugiere una tipología triple de economías del conocimiento y sociedades del conocimiento que exige revisiones en el enfoque tradicionalmente binario del análisis de los modelos de competitividad económica y cohesión social. Más que centrarnos únicamente en las diferencias entre los modelos de mercado neoliberales y sociales de la economía del conocimiento, con las deducciones políticas típicas de los planteamientos de suma cero entre políticas y características asociadas a cada uno 
de ellos, deberíamos suponer que existen por lo menos tres modelos de economía del conocimiento o de la sociedad del conocimiento en Europa, y que el modelo nórdico es, de hecho, bastante diferente del modelo de mercado social de los estados centroeuropeos, con una mayor capacidad para combinar los beneficios de la competitividad económica y la cohesión social que el modelo centroeuropeo o el modelo neoliberal. En este sentido, los países nórdicos se aproximan más al objetivo de Lisboa que cualquiera de los otros dos grupos, y más de lo que se ha reconocido actualmente en los debates políticos sobre el futuro de Europa.

Si este es el caso, ello tiene importantes repercusiones para la política de formación a lo largo de toda la vida. Los debates europeos suponen con razón que la creciente competitividad europea requiere mejorar los índices de empleo (Kok, 2004); también reconocen, aunque con un énfasis cada vez menor, que mantener la cohesión social requiere no sólo la inclusión social a través del empleo, sino también niveles razonables de igualdad en los ingresos. Como muestran los ejemplos nórdicos, la formación a lo largo de toda la vida puede tener un papel vital en conseguir ambos objetivos. Sin embargo, sólo podrá hacerse si se reconoce, más de lo que sucede actualmente, que incrementar la igualdad en la educación importa tanto como elevar los estándares generales.

\section{Referencias bibliográficas}

Albert, M. (1993). Capitalism against Capitalism. Londres: Whurr Publishers.

Blanden, J.; GregG, P. y MACHIN, S. (2005). Intergenerational Mobility in Europe and North America. Londres: Sutton Trust.

Boucher, L. (1982). Tradition and Change in Swedish Education. Oxford: Pergamon Press.

Brown, P.; Green, A. y Lauder, H. (2001). High Skills: Globalization, Competitiveness and Skills Formation. Oxford: Oxford University Press.

Crouch, C.; Finegold, D. y Sato, M. (1999). Are Skills the Answer?: The Political Economy of Skill Creation in Advanced Industrial Countries. Oxford: Oxford University Press.

Crouch, C. y Streeck, W. (2006). The Diversity of Democracy: Corporatism, Social Order and Political Conflict. Cheltenham: Edward Elgar.

Dore, R. (2000). Stock Market Capitalism: Welfare Capitalism, Japan and Germany Versus the Anglo-Saxons. Oxford: Oxford University Press.

EsPing-ANDERSEN, G. (1990). The Three Worlds of Welfare Capitalism. Cambridge: Polity.

- (2003). «Unequal Opportunities and Social Inheritance». En: CoraK, M. (ed.). The Dynamics of Intergenerational Income Mobility. Cambridge: Cambridge University Press.

European Commission (EC) (1999). Continuing Training in Enterprises: Facts and Figures. European Training Youth. Brussels: EC.

FineGold, D. y SOskiCE, S. (1988). «The Failure of Training in Brtain: Analysis and Prescriptions». Oxford Review of Economic Policy, 4 (3), 21-53.

Green, A. y Preston, J. (2001). «Education and Social Cohesion: Re-centering the Debate». Peabody Journal of Education, 76(3 y 4), 247-284.E 
Green, A.; Preston, J. y Janmaat, G. (2006). Education, Equality and Social Cohesion. Londres: Palgrave.

Green, A. y Janmaat, J.G. (2011). Regimes of Social Cohesion. Societies and the Crisis of Globalisation. Basingstoke: Palgrave

Green, A., Preston, J. y Janmaat, J.G. (2006) Education, Equality and Social Cohesion. Basingstoke: Palgrave.

Hall, P. y Soskice, D. (eds.) (2001). Varieties of Capitalism: The Institutional Foundations of Comparative Advantage. Oxford: Oxford University Press.

Hutton, W. (1995). The State We're In. Kent: Mackays of Chatham.

- (2002). The World We're In. Londres: Little, Brown.

The KOK REPORT (2004). Facing the Challenge: The Lisbon Strategy for Growth and Employment (refrendado por los jefes de Estado de la UE y la Comisión Europea en noviembre de 2004). Comisión Europea.

Leney, T.; Ammerman, P.; Brandsma, J.; Behringer, F.; Coles, M.; Feenstra, B.; Grollman, P.; Green, A.; Shapiro, H. and Westerhuis, A. (2004). Achieving the Lisbon Goal: The Contribution of VET. Report to the European Commission. London: Qualification and Curriculum Authority (QCA).

Lloyd, C. y PAYNE, J. (2004). "Idle Fancy" or "Concrete Will?»: Defining and Realising a High Skills Vision for the UK, SKOPE Research Paper No. 47. Warwick: SKOPE, Universidad de Warwick.

Mooij, R. de y TAnG, P (2003). Four Futures of Europe. La Haya: Centraal Planbureau.

Nickel, S. and Layard, R. (1998). Labour Market Institutions and Economic Performance. Londres: Centre for Economic Performance, LSE.

OCDE (2000). Literacy in the Information Age. París: OCDE.

OCDE (2002). Reading for a Change: Performance and Engagement Across Countries. Results from PISA 2000. París: OCDE.

OCDE (2004). Social Expenditure Database (SOCX). (www.oecd.org/els/social/expenditure).

OCDE (2007). PISA 2006: Science Competencies for Tomorrow's World. Volumes 1 and 2. Paris: OCDE

Rubenson, K. (2002). The Nordic Model of Adult Education. Vancouver: Centre for Higher Education Research, University of British Columbia.

StreecK, W. (1992). Social Institutions and Economic Performance. Londres: Sage.

- (1997). "German Capitalism: Does it Exist? Can it Survive?». New Political Economy, 2 (2), 237-256.

StreecK, W. y SchmitTer, P. (1991). «From National Corporatism to Transnational Pluralism: Organised Interests in the Single European Market». Politics and Society, 19 (2), 133-164.

World Bank (2001). World Development Report: Attacking Poverty. Oxford: Oxford University Press. 